\title{
Conditional Optimization and One Inverse Boundary Value Problem
}

\author{
Pyotr N. Ivanshin \\ Physics Institute, Kazan Federal University, Kremlyovskaya Street 18, Kazan 420008, Russia \\ Correspondence should be addressed to Pyotr N. Ivanshin; pivanshi@yandex.ru
}

Received 20 April 2015; Accepted 23 June 2015

Academic Editor: Debasish Roy

Copyright ( 2015 Pyotr N. Ivanshin. This is an open access article distributed under the Creative Commons Attribution License, which permits unrestricted use, distribution, and reproduction in any medium, provided the original work is properly cited.

\begin{abstract}
Here we construct different approximate solutions of the plane inverse boundary value problem of aerohydrodynamics. In order to do this we solve some conditional optimization problems in the norms $\|\cdot\|_{2},\|\cdot\|_{\infty}$, and $\|\cdot\|_{1}$ and some of their generalizations. We present the example clarifying the mathematical constructions and show that the supremum norm generalization seems to be the optimal one of all the functionals considered in the paper.
\end{abstract}

\section{Introduction}

The formulation and the first attempt in solution of the inverse boundary value problem of aerohydrodynamics are due to Tumashev and Mangler $[1,2]$.

This problem is one of numerous boundary value problems. The example of the boundary value problem is the Hilbert one: given the real value of the analytic function on the known contour $C$ it should be possible to reconstruct this function in the inner domain with respect to the contour $C$ [3].

The example of the inverse boundary value problem is the following: Assume that we have two real functions $u(s), v(s)$, $s \in[0, l]$, such that $u(0)=u(l)$ and $v(0)=v(l)$. It should be possible to reconstruct both the contour $C$ of length $l$ and the analytic in the domain bounded by $C$ function whose real and imaginary parts at the contour $C$ point with the arc parameter $s$ coincide with the given functions $u(s), v(s)$ [2].

The inverse boundary value problem solution methods can be applied for problems of aerohydrodynamics [2, 4]. For example, all the wing construction methods I know of reduce to two basic problems [5]. The first of them is the plane wing section reconstruction, and the second is mutual positioning of these profiles. We consider the former of these problems.

Our problem is as follows: we have the air or fluid flow particles velocity distribution along the length of the unknown contour $C$, that is, the function $v(s), s \in[0, l]$. So we know only the contour $C$ length $l$ and not the form of this profile. We also assume that the air or fluid flow is potential.
The inverse boundary value problem then is to find the shape of the contour $C$ depending only on the information on the particles velocity. Since the velocity is given only in the finite set of points it seems natural to apply a spline (not necessarily linear) in order to define this function for all the contour points. This approach was widely used by the authors of [4].

This problem requires certain resolution conditions which appear only after we find the contour parametrization. So these conditions cannot be satisfied initially. Here we try to find a minimal in some sense initial data deformation so that this deformed data meets these conditions. Note that almost all of the equation systems in the article can be solved only numerically due to their transcendent form.

Let us recall the solution procedure from [2].

(1) Inverse Boundary Value Problem Solution. Let the velocity distribution $v(s)$ along the unknown contour $C$ be given; here $s$ is the curve length parameter. We must find the contour $C$ form. Assume that the contour length equals $l$, the flow bifurcation happens at the point with the parameter value $s_{a} \in(0, l)$, and the flow velocity vanishes at $s=0, s=l$. Let $v(s)<0$ for $s \in\left[0, s_{a}\right)$ and $v(s)>0$ for $s \in\left(s_{a}, l\right]$.

We consider the constants

$$
\begin{aligned}
\phi_{h} & =\int_{0}^{s_{a}} v(s) d s, \\
\phi_{l} & =\int_{s_{a}}^{l} v(s) d s
\end{aligned}
$$


and the function

$$
\phi(s)= \begin{cases}\int_{0}^{s} v(t) d t, & s \in\left[0, s_{a}\right] \\ \phi_{l}-\int_{s_{a}}^{s} v(t) d t, & s \in\left[s_{a}, l\right] .\end{cases}
$$

Here the number $\phi_{h}-\phi_{l}$ is the flow circulation. If it is greater than 0 then we have a positive lifting force for the wing profile.

Now we have the standard external inverse boundary value problem for the complex potential $\omega=\phi+i \psi$ in the unknown contour $C$ exterior. This function $\omega$ has a simple pole and a logarithmic singularity at infinity and meets the boundary conditions

$$
\begin{aligned}
& \phi=\phi(s), \\
& \psi \equiv 0 .
\end{aligned}
$$

We introduce the auxiliary analytic function. First note that the complex potential of the flow over the unit disc with the circulation equal to $\phi_{h}-\phi_{l}$ has the following form:

$$
\omega=w(\zeta)=-U_{0}\left(\zeta+\frac{1}{\zeta}\right)+\frac{\phi_{h}-\phi_{l}}{2 \pi i} \ln \zeta+C^{*} .
$$

Here we have the relations $\phi=\operatorname{Re}(\omega)=-2 U_{0} \cos \gamma+\left(\left(\phi_{h}-\right.\right.$ $\left.\left.\phi_{l}\right) / 2 \pi\right) \gamma+C^{*}$ and $v=d \phi / d \gamma=2 U_{0} \sin \gamma+\left(\phi_{h}-\phi_{l}\right) / 2 \pi$ for $\zeta=e^{i \gamma}$.

Since the velocity is 0 in the critical points (where flow separates into two parts and where it vanishes) we have the following equations on the unknown constants $U_{0}, C^{*}$ and two auxiliary angles relative to the flow critical points $\gamma_{1}$ and $\gamma_{2}$ :

$$
\begin{aligned}
-2 U_{0} \cos \gamma_{1}+\frac{\phi_{h}-\phi_{l}}{2 \pi} \gamma_{1}+C^{*} & =0, \\
-2 U_{0} \cos \gamma_{2}+\frac{\phi_{h}-\phi_{l}}{2 \pi} \gamma_{2}+C^{*} & =\phi_{l}, \\
2 U_{0} \sin \gamma_{1}+\frac{\phi_{h}-\phi_{l}}{2 \pi} & =0, \\
2 U_{0} \sin \gamma_{2}+\frac{\phi_{h}-\phi_{l}}{2 \pi} & =0 .
\end{aligned}
$$

This system is uniquely resolvable [2].

Now we equate the complex flow potential real parts of the contour $C$ in the complex plane of coordinate $z=x+i y$ to the complex flow potential of the unit circle in the plane of coordinate $\zeta=r e^{i \gamma}, r \geq 0$ :

$$
\phi(s)=\operatorname{Re} w(\gamma) .
$$

Then we obtain the relation $s=s(\gamma)$ on the parameters of the contours. Finally we reconstruct the function $z=z(\zeta)$, which maps the unit disc exterior onto the flow domain in the plane $z$ solving the Schwartz problem for the function $\ln z^{\prime}(\zeta)$ at the unit disc exterior. The main object for our consideration is now the function

$$
v(\gamma)=\frac{d s}{d \gamma}=\frac{d \operatorname{Re}(w(\gamma))}{d \gamma}: \frac{d \phi}{d s} .
$$

(2) Resolvability Conditions. The profile possesses the mechanical sense in case the function $v(\gamma)$ of the previous section meets certain conditions.

The first condition on the function $\log |v(\gamma)|$ naturally appears when we reconstruct our contour and equate the residue of $\log |v(\gamma)|$ at infinity to 0 . Then the profile is a closed curve.

So the contour $C$ closeness condition is as follows: the function $\log |v(\gamma)|$ must meet the complex equality

$$
\int_{0}^{2 \pi} \log |v(\gamma)| e^{i \gamma} d \gamma=A+i B
$$

Here $A$ and $B$ are known constants. Thus the first condition defines the first Fourier coefficients with $\cos \gamma$ and $\sin \gamma$ for the function $\log |v(\gamma)|$.

We arrive at the second condition on $\log |v(\gamma)|$ due to purely mechanical reasons. Since the flow velocity is fixed at infinity (this is a flight speed) we equate the value of $\log |v(\gamma)|$ at infinity to some constant and obtain the following condition on $\log |v(\gamma)|$ :

$$
\int_{0}^{2 \pi} \log |v(\gamma)| d \gamma=C
$$

Note that the constants $A, B$, and $C$ values under conditions (8), (9) depend only on the form of Zhukovskii-Mitchell modified function $[2,4]$.

So in the general case, that is, when the conditions do not hold, the problem does not have a solution and becomes an ill-posed problem.

(3) The Mathematical Problem. Ivanov [6] proposed application of quasisolutions to this problem. The notion of "quasisolution" was introduced earlier by Elizarov and Fokin $[7,8]$ and in our case can be described as follows.

Definition 1. Let the inverse boundary problem with the given velocity $v_{\infty}$ resolution procedure result in the function $\log |v(\gamma)|$ such that its first Fourier coefficients do not equal the desired ones. Let one denote the set of functions with appropriate first Fourier coefficients by $U$. Then one says that quasisolution of the problem is the aerodynamic contour for whose construction we apply some function $q_{0}(\gamma) \in U \subset P$ instead of $\log |v(\gamma)|$. Here one chooses $q_{0}(\gamma)$ in the normed space $P$ so that

$$
\left\|\log |v(\gamma)|-q_{0}(\gamma)\right\|_{P}=\min _{q \in P}\|\log |v(\gamma)|-q(\gamma)\|_{P} .
$$

Thus we need to modify the function $\log |v(\gamma)|$ so that this modified function meets conditions (8), (9). Naturally this modification affects the initial data $v(s)$. So we must change the initial velocity distribution in some way.

This modification is as follows.

We consider the function $\log |v(\gamma) F(\gamma)|$ instead of the constructed $\log |v(\gamma)|$. Assume that $\log |v(\gamma) F(\gamma)|$ meets conditions (8), (9). Clearly we need to make the $\log |v(\gamma)|$ 
modification as small as possible; that is, we search for the function $\log |F(\gamma)|$ with the minimal possible norm.

In [6] one can find the quasisolution which in our notation minimizes the function $\log |F(\gamma)|$ norm in $L^{2}[0,2 \pi]$. The solution procedure of [6] gave us $\log |\tilde{f}(\gamma)|$ in the form of the Fourier polynomial which allowed $\log v(\gamma)$ to meet the desired conditions.

It seems necessary to note that the articles $[3,9]$ contain function minimization similar to one presented here with respect to the norm $\|\cdot\|_{\infty}$. At the same time both the posed problems and the solution technique differ from that given in this paper.

We have purely mathematical problem.

We search for the function $F(\gamma)$ minimal in some norm under the following restrictions on its Fourier coefficients:

$$
\begin{gathered}
\int_{0}^{2 \pi} F(\gamma) d \gamma=C-\int_{0}^{2 \pi} \ln v(\gamma) d \gamma=C^{\prime}, \\
\int_{0}^{2 \pi} F(\gamma) \cos \gamma d \gamma=A-\int_{0}^{2 \pi} \ln v(\gamma) \cos \gamma d \gamma=A^{\prime}, \\
\int_{0}^{2 \pi} F(\gamma) \sin \gamma d \gamma=B-\int_{0}^{2 \pi} \ln v(\gamma) \sin \gamma d \gamma=B^{\prime} .
\end{gathered}
$$

We preserve the former of these relations and we rewrite the two latter relations as follows:

$$
\begin{aligned}
& \int_{0}^{2 \pi} F(\gamma) \sin (\gamma+\beta) d \gamma=K \\
& \int_{0}^{2 \pi} F(\gamma) \cos (\gamma+\beta) d \gamma=0
\end{aligned}
$$

and here $K=\left|A^{\prime}+i B^{\prime}\right|, \beta=\pi / 2-\arg \left(A^{\prime}+i B^{\prime}\right)$.

Without loss of generality we assume that $\beta=0$. In the other case we simply consider the shifted variable $t=\gamma+\beta$ instead of the initially given $\gamma$.

Finally we have the following problem.

We search for the function $F(t)$ minimal in some norm under the following three conditions:

$$
\begin{aligned}
\int_{0}^{2 \pi} F(t) d t & =C^{\prime} \\
\int_{0}^{2 \pi} F(t) \sin t d t & =K>0 \\
\int_{0}^{2 \pi} F(t) \cos t d t & =0 .
\end{aligned}
$$

Here we construct the functions $F(t)$ which minimize functionals $\|\log |F(t)|\|_{\infty},\left\|\log \left|F^{(n)}(t)\right|\right\|_{1}$, and $\|\log |F(t)|\|_{\infty}+\lambda\left\|\log \left|F(t)^{\prime}\right|\right\|_{\infty}, \lambda>0$, and give the solution for $L^{p}[0,2 \pi]$ norm. We also present the example that compares the approximate solutions constructed with the help of the norms $\|\log |F(t)|\|_{\infty}+1 / 2\left\|\log \left|F(t)^{\prime}\right|\right\|_{\infty},\|F(t)\|_{2}$ and the functional $\int_{0}^{2 \pi}\left|F^{3}(t)\right| d t$.

\section{Approximate Solution Which Minimizes $\|F\|_{\infty}$}

The results of the section were proved in [10].

2.1. Conditions (13): Modification Function Construction. Let us first solve the auxiliary problem. Given the function $q$ : $[-\pi, \pi] \rightarrow \mathbb{R}$ it is possible to construct the class $M_{q}$ of functions $F:[-\pi, \pi] \rightarrow \mathbb{R}$ which meet the condition

$$
\int_{-\pi}^{\pi} F(t) q(t) d t=K>0 .
$$

We must find $\min _{M_{q}}\|F\|_{\infty}$.

Clearly the possible solution space choice yields the following result:

$$
F_{0}(t)= \begin{cases}K T, & q(t) \geq 0 \\ -K T, & q(t)<0\end{cases}
$$

and here $T=1 / \int_{-\pi}^{\pi}|q(t)| d t$. This can be easily proved by assuming the negative. Indeed, let us take into consideration a function $h:[-\pi, \pi] \rightarrow \mathbb{R}$ such that $\left\|F_{0}+h\right\|_{\infty}<\left\|F_{0}\right\|_{\infty}$. Then $h(t)<0$ for $q(t)>0$ and $h(t)>0$ for $q(t)<0$. At the same time $\int_{-\pi}^{\pi} h(t) q(t) d t>0$. Hence $\int_{-\pi}^{\pi}\left(F_{0}(t)+h(t)\right) q(t) d t>K$, and this inequality contradicts condition (14).

Let us apply this auxiliary problem solution to the case of $q(t)=\sin t$. Then

$$
F_{0}(t)= \begin{cases}\frac{K}{4}, & t \in(0, \pi) \\ -\frac{K}{4}, & t \in(-\pi, 0) .\end{cases}
$$

Note that $\int_{-\pi}^{\pi} F_{0}(t) \cos t d t=0$. Thus under conditions (13) the auxiliary function $f(\gamma)$ can be found from relation $\ln (d f / d \gamma)=F_{0}(\gamma)$.

Let $U$ be the set of functions integrable on $[0,2 \pi]$ meeting conditions (13). Given these conditions it should be possible to find in $U$ the extreme function $F_{1}(t)$ such that

$$
\left\|F_{1}\right\|_{\infty}=\min _{U}\|F\|_{\infty} .
$$

Clearly the function $\check{F}(t) \equiv C^{\prime} / 2 \pi$ is the solution of the problem for $K=0$, and $F_{0}(t)$ is also the solution for the case of $C^{\prime}=0$. Thus it feels natural to construct the solution similarly to [4] and consider the sum $\check{F}(t)+F_{0}(t)$. Nevertheless the solution minimizing the norm $\|F\|_{\infty}$ under condition of nonzero $C^{\prime}$ and $K$ differs from the given sum.

Let us find now the constants $\varepsilon>0$ and $\alpha \in[0, \pi / 2]$ so that

$$
\begin{aligned}
& \varepsilon \int_{0}^{\pi+\alpha} d t-\varepsilon \int_{\pi+\alpha}^{2 \pi-\alpha} d t+\varepsilon \int_{2 \pi-\alpha}^{2 \pi} d t=C^{\prime} \\
& \varepsilon \int_{0}^{\pi+\alpha} \sin (t) d t-\varepsilon \int_{\pi+\alpha}^{2 \pi-\alpha} \sin (t) d t+\varepsilon \int_{2 \pi-\alpha}^{2 \pi} \sin (t) d t \\
& \quad=K
\end{aligned}
$$


that is, we search for the solution of the equation system

$$
\begin{aligned}
4 \varepsilon \alpha & =C^{\prime}, \\
4 \varepsilon \cos (\alpha) & =K .
\end{aligned}
$$

This nonlinear system has a unique solution. The constant $\alpha$ value is the solution of equation $\cos (\alpha) / \alpha=K / C^{\prime}$. This relation possesses a unique solution in the interval $(0, \pi / 2)$. After we obtain the value of $\alpha$ we naturally determine the one and only value of $\varepsilon>0$.

Thus existence of $\varepsilon$ and $\alpha$ makes it possible to construct the desired function

$$
F_{1}(t)= \begin{cases}\varepsilon, & t \in[0, \pi+\alpha] \bigcup[2 \pi-\alpha, 2 \pi] \\ -\varepsilon, & t \in(\pi+\alpha, 2 \pi-\alpha)\end{cases}
$$

It is easy to verify that the function $F_{1}(t)$ meets the last condition of (13).

Proposition 2. Piecewise constant function of relation (20) is the problem solution for $C^{\prime}>0$.

2.2. Quasisolution Which Minimizes $\|F\|_{\infty}+\lambda\left\|F^{\prime}\right\|_{\infty}$. Let $M$ be the set of functions $F:[-\pi, \pi] \rightarrow \mathbb{R}$ with almost every where bounded first derivative, meeting conditions (13). Given these conditions it should be possible to find a function minimizing the functional

$$
\min _{M}\left(\|F\|_{\infty}+\lambda\left\|F^{\prime}\right\|_{\infty}\right)
$$

Function class choice makes us search for the function which meets the equations $\lambda f^{\prime}(t) \pm f(t) \equiv$ const in subsets of the interval $[0,2 \pi]$. Solutions of these equations are the functions $k_{1}-k_{2} e^{ \pm(1 / \lambda) t}$. The constants $k_{1}$ and $k_{2}$ must be chosen so that the solution is the continuous $2 \pi$-periodic function on $\mathbb{R}$.

For example, for $\lambda=1 / 2$ we obtain the following solution:

$$
f(t)= \begin{cases}c\left(1-e^{-2 t}\right), & t \in\left[0, \frac{\pi}{2}\right] \\ c\left(1-e^{2(t-\pi)}\right), & t \in\left[\frac{\pi}{2}, \pi\right] \\ c\left(e^{-2(t-\pi)}-1\right), & t \in\left[\pi, \frac{3 \pi}{2}\right] \\ c\left(e^{2(t-2 \pi)}-1\right), & t \in\left[\frac{3 \pi}{2}, 2 \pi\right]\end{cases}
$$

and here $c=5 K / 8\left(2+e^{-\pi}\right)$ can be found from conditions (13).

2.2.1. Conditions (13) Satisfaction. Let us for the sake of simplicity fix $\lambda=1 / 2$.

2.2.2. Problem Formulation. Let $T$ be a subclass of $2 \pi$ periodic functions which meet conditions (13). Find the function $f_{0}(t) \in T \subset W^{1, \infty}[0,2 \pi]$ such that

$$
\left\|f_{0}\right\|_{\infty}+\frac{1}{2}\left\|f_{0}^{\prime}\right\|_{\infty}=\min _{T}\left(\|f\|_{\infty}+\frac{1}{2}\left\|f^{\prime}\right\|_{\infty}\right) \text {. }
$$

Let us construct the piecewise smooth function glued from the functions of the form $\pm c\left(1-e^{ \pm 2 t}\right)$.

For the case of

$$
\frac{4 C^{\prime}}{5 K}<\frac{2 \pi-1+e^{-2 \pi}}{1+e^{-2 \pi}} \approx 5.2752
$$

we consider the function

$$
f(t)= \begin{cases}c\left(1-e^{-2(t+\alpha)}\right), & t \in\left[-\alpha, \frac{\pi}{2}\right] ; \\ c\left(1-e^{2(t-\pi-\alpha)}\right), & t \in\left[\frac{\pi}{2}, \pi+\alpha\right] ; \\ c\left(e^{-2(t-\pi-\alpha)}-1\right), & t \in\left[\pi+\alpha, \frac{3 \pi}{2}\right] ; \\ c\left(e^{2(t-2 \pi+\alpha)}-1\right), & t \in\left[\frac{3 \pi}{2}, 2 \pi-\alpha\right] .\end{cases}
$$

The unknown variables $c$ and $\alpha$ are the solutions of the system

$$
\begin{aligned}
2 c\left(2 \alpha-e^{-\pi} \sinh (2 \alpha)\right) & =C^{\prime}, \\
\frac{8}{5} c\left(2 \cos (\alpha)+e^{-\pi} \cosh (2 \alpha)\right) & =K .
\end{aligned}
$$

In particular $\alpha$ is the solution of the equation

$$
\frac{\left(2 \alpha-e^{-\pi} \sinh (2 \alpha)\right)}{2 \cos (\alpha)+e^{-\pi} \cosh (2 \alpha)}=\frac{4 C^{\prime}}{5 K} .
$$

Under condition (24) this equation has a unique solution in the interval $(0, \pi / 2)$.

Proposition 3. The function given by relation (25) is the solution of the problem under condition (24).

Let us now consider the case of false relation (24). Then the solution is the function

$$
f(t)= \begin{cases}c\left(1-e^{-2(t+a)}\right), & t \in\left[-\frac{\pi}{2}, \frac{\pi}{2}\right] ; \\ c\left(1-e^{2(t-\pi-a)}\right), & t \in\left[\frac{\pi}{2}, \frac{3 \pi}{2}\right] .\end{cases}
$$

Here the system on $a$ and $c$ is as follows:

$$
\begin{aligned}
c\left(2 \pi-2 \sinh (\pi) e^{-2 a}\right) & =C^{\prime}, \\
c \frac{8}{5} \cosh (\pi) e^{-2 a} & =K .
\end{aligned}
$$

This system is uniquely resolvable for $a \geq \pi / 2$. Clearly for $K \rightarrow 0$ we have $a \rightarrow+\infty$ and $f(t) \equiv c$.

Proposition 4. The function given by relation (28) solves the problem for the case of

$$
\frac{4 C^{\prime}}{5 K} \geq \frac{2 \pi-1+e^{-2 \pi}}{1+e^{-2 \pi}} .
$$

Note that the constant $\lambda$ describes the relative impact of the auxiliary function derivative on the approximate solution. The greater this constant is the closer the resulting minimizing function is to a piecewise-linear curve being the solution of the similar problem for the functional $\left\|F^{\prime}\right\|_{\infty}$ (cf. Figure 1). 


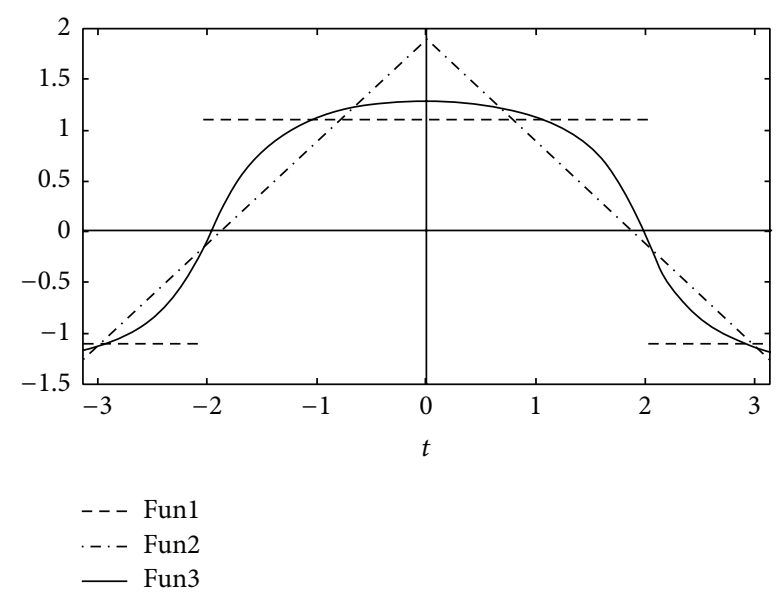

FIGURE 1: Fun1: the solution corresponding to the norm $\|F\|_{\infty},(\lambda=$ $0)$. Fun2: the solution corresponding to the functional $\left\|F^{\prime}\right\|_{\infty},(\lambda \rightarrow$ $\infty)$. Fun3: the solution corresponding to the norm $\|F\|_{\infty}+1 / 2\left\|F^{\prime}\right\|_{\infty}$.

\section{Approximate Solution Minimizing $\|F\|_{L_{1}}$}

The results of the section were given without proof in [11].

Here we solve the problem for the integral norm $\|f\|_{1}=$ $\int_{-\pi}^{\pi}|f(t)| d t$. We obtain the solution for the norm opposite to the norm $\|\cdot\|_{\infty}$ on the spaces $L^{p}$ scale.

Since the solution exists only in the space of distributions (generalized functions) [12] it cannot be immediately applied to mechanical problems. Indeed let us first find the function with minimal possible norm $\|F\|_{L^{1}}$ meeting the condition

$$
\int_{-\pi}^{\pi} f(t) g(t) d t=K
$$

It is clear that the solution is a function consisting of summands of type $\operatorname{sgn}(g(t)) K \delta_{x_{0}}(t)$, and here $g\left(x_{0}\right)=$ $\max \{|g(t)| \mid t \in[-\pi, \pi]\}$. For example, for the function $g(t)=$ $\sin (t)$ we obtain the extremal $F(t)=\left(\delta_{\pi / 2}(t)-\delta_{3 \pi / 2}(t)\right) / 2$. Here $\delta_{x}(t)$ is a Dirac $\delta$-function [12]. At the same time $\alpha \delta_{x}(t)$ must be considered as a limit of a function sequence $q_{n}(t)$ $\operatorname{such}$ that $\operatorname{supp}\left(f_{n}\right) \rightarrow x$ and $\int_{0}^{2 \pi} q_{n}(t) d t=\alpha$.

3.1. Problem Formulation. Let us find in the set of all integrable on $[0,2 \pi]$ distributions meeting conditions (13), one with the minimal possible norm $\|f\|_{L^{1}}$.

3.2. Problem Solution. The first two solutions presented here are of purely mathematical interest since due to their noncontinuity they cannot be applied for any actual profile reconstruction.

\section{Proposition 5. The function}

$$
f_{0}(t)=\frac{C^{\prime}+K}{2} \delta_{\pi / 2}(t)+\frac{C-K}{2} \delta_{3 \pi / 2}(t)
$$

solves the posed problem.

The proof of this statement is similar to the one of [11].
Proof. Assume that there exists a function $g(t)$ such that $\int_{0}^{2 \pi}\left|f_{0}(t)+g(t)\right| d t<\int_{0}^{2 \pi}\left|f_{0}(t)\right| d t=\max \left\{\left|C^{\prime}\right|, K\right\}$, $\int_{0}^{2 \pi} g(t) d t=0$, and $\int_{0}^{2 \pi} g(t) \sin (t) d t=0$.

We first show that $g(t)=\varepsilon_{1} \delta_{\pi / 2}(t)+\varepsilon_{2} \delta_{3 \pi / 2}(t)$. Assume the contrary. Let $g(t)$ be an integrable bounded function. Hence $g(t)$ does not possess summands of $\delta$-function type. Then

$$
\begin{aligned}
\int_{0}^{2 \pi}\left|f_{0}(t)+g(t)\right| d t & =\int_{0}^{2 \pi}\left|f_{0}(t)\right| d t+\int_{0}^{2 \pi}|g(t)| \\
& >\int_{0}^{2 \pi}\left|f_{0}(t)\right| d t .
\end{aligned}
$$

This contradicts the assumption. So $g(t)=\alpha \delta_{\pi / 2}(t)+$ $\beta \delta_{3 \pi / 2}(t)+h(t)$, and here $h(t)$ does not have summands in the form of $\delta_{\pi / 2}(t)$ and $\delta_{3 \pi / 2}(t)$ with some multipliers.

Assume first that $h(t)$ is not a distribution (i.e., it does not contain $\delta$-summands). Then

$$
\begin{aligned}
\int_{0}^{2 \pi}\left|f_{0}(t)+g(t)\right| d t= & \left|\frac{C^{\prime}+K}{2}+\varepsilon_{1}\right| \\
& +\left|\frac{C^{\prime}-K}{2}+\varepsilon_{2}\right| \\
& +\int_{0}^{2 \pi}|h(t)| d t \\
& >\int_{0}^{2 \pi}\left|f_{0}(t)+g(t)-h(t)\right| d t .
\end{aligned}
$$

Thus we may assume that $h(t)=0, \forall t \in[0,2 \pi]$.

Assume now that $h(t)=\sum_{k=3}^{\infty} \varepsilon_{k} t_{k}$. Then by assumption we obtain the relation

$$
\begin{aligned}
& \sum_{k=3}^{\infty}\left|\varepsilon_{k}\right|+\left|\frac{C^{\prime}+K}{2}+\varepsilon_{1}\right|+\left|\frac{C^{\prime}-K}{2}+\varepsilon_{2}\right| \\
& <\max \left\{\left|C^{\prime}\right|, K\right\} .
\end{aligned}
$$

The following also holds true:

$$
\begin{array}{r}
\varepsilon_{1}+\varepsilon_{2}+\sum_{k=3}^{\infty} \varepsilon_{k}=0, \\
\varepsilon_{1}-\varepsilon_{2}+\sum_{k=3}^{\infty} \varepsilon_{k} \sin \left(t_{k}\right)=0 .
\end{array}
$$

Let $C^{\prime}>K$. Then the first relation of (13) yields $\varepsilon_{1}+\varepsilon_{2}<$ $-\sum_{k=3}^{\infty}\left|\varepsilon_{k}\right|$. So the first relation is false. If $K>C^{\prime}$ the second relation of (13) is violated. For $C^{\prime}=K$ we have $\varepsilon_{2}=0$ and violation of both of the relations. Hence $h(t)$ does not have summands in the form of $\varepsilon_{k} \delta_{t_{k}}(t), t_{k} \neq \pi / 2,3 \pi / 2$.

Let us now prove that $\varepsilon_{1} \stackrel{\delta_{k}}{=} \varepsilon_{2}=0$. The second relation $\left(\int_{0}^{2 \pi} g(t) d t=0\right)$ implies that $\varepsilon_{1}+\varepsilon_{2}=0$. The third relation $\left(\int_{0}^{2 \pi} g(t) \sin (t) d t=0\right)$ in its turn tells us that $\varepsilon_{1}-\varepsilon_{2}=0$. So $\varepsilon_{1}=\varepsilon_{2}=0$. 
By direct calculation we obtain

$$
\int_{0}^{2 \pi} f_{0}(t) \cos (t) d t=0 .
$$

Consider now the functional

$$
L_{1,1}(f)=\int_{0}^{2 \pi}\left|f^{\prime}(t)\right| d t .
$$

Let us find the function meeting conditions (13) and minimizing $L_{1,1}(f)$.

Proposition 6. Piecewise constant function of (20) solves the problem.

Let us first give the direct proof.

Proof. Let there exist a function $G_{1}$ meeting conditions (13) and such that $L_{1,1}\left(G_{1}\right)<L_{1,1}\left(F_{1}\right)$.

(1) If $G_{1}-F_{1}$ is continuous then $L_{1,1}\left(G_{1}-F_{1}+F_{1}\right)=$ $L_{1,1}\left(G_{1}-F_{1}\right)+L_{1,1}\left(F_{1}\right)>L_{1,1}\left(F_{1}\right)$.

(2) Since $G_{1}$ again can be considered to be symmetric with respect to $t=\pi / 2$ its points of discontinuity are also symmetrically positioned with respect to $t=\pi / 2$. Hence $G_{1}=H_{1}+J_{1}$, and here $H_{1}$ is piecewise constant and $J_{1}$ is continuous. Item yields $L_{1,1}\left(H_{1}\right) \leq L_{1,1}\left(G_{1}\right)$.

(3) Let $g=H_{1}-H_{1}$ be a simple piecewise constant function:

$$
g=\sum_{n} a_{n} \chi_{\left[x_{n}, y_{n}\right]}
$$

(4) Note that the first condition of (13) implies that the function $g$ can be represented as $\sum_{n}\left(b_{n} \chi_{\left[x_{n}, y_{n}\right]}-\right.$ $\left.c_{n} \chi_{\left[x_{n}^{\prime}, y_{n}^{\prime}\right]}\right)$; moreover $b_{n}\left(y_{n}-x_{n}\right)-c_{n}\left(y_{n}^{\prime}-x_{n}^{\prime}\right)=0$ and $b_{n}, c_{n}>0$.

(5) Consider the relations

$$
\begin{aligned}
P_{1}= & b_{n}\left(y_{n}-x_{n}\right)-c_{n}\left(y_{n}^{\prime}-x_{n}^{\prime}\right), \\
P_{2}= & b_{n} \sin \left(\frac{y_{n}+x_{n}}{2}\right) \sin \left(\frac{y_{n}-x_{n}}{2}\right) \\
& -c_{n} \sin \left(\frac{y_{n}^{\prime}+x_{n}^{\prime}}{2}\right) \sin \left(\frac{y_{n}^{\prime}-x_{n}^{\prime}}{2}\right) .
\end{aligned}
$$

(6) Note that, for $b_{n}, c_{n} \neq 0, P_{1}>P_{2}$. It is also clear that $\left[x_{n}, y_{n}\right] \in[\pi+\alpha, 2 \pi-\alpha]$.

There exist two possibilities:

(1) $\left[x_{n}^{\prime}, y_{n}^{\prime}\right] \in[0, \pi]$,

(2) $\left[x_{n}^{\prime}, y_{n}^{\prime}\right] \in[\pi, \pi+\alpha]$.

In case of (1) we have $b_{n} \sin \left(\left(y_{n}+x_{n}\right) / 2\right) \sin \left(\left(y_{n}-x_{n}\right) / 2\right)<$ 0 and $-c_{n} \sin \left(\left(y_{n}^{\prime}+x_{n}^{\prime}\right) / 2\right) \sin \left(\left(y_{n}^{\prime}-x_{n}^{\prime}\right) / 2\right)<0$.
In case of (2) we have $b_{n} \sin \left(\left(y_{n}+x_{n}\right) / 2\right) \sin \left(\left(y_{n}-x_{n}\right) / 2\right)>$ 0 , but $-c_{n} \sin \left(\left(y_{n}^{\prime}+x_{n}^{\prime}\right) / 2\right) \sin \left(\left(y_{n}^{\prime}-x_{n}^{\prime}\right) / 2\right)<0$, but the integral mean theorem yields

$$
\begin{aligned}
& \left|\frac{\sin \left(\left(y_{n}+x_{n}\right) / 2\right) \sin \left(\left(y_{n}-x_{n}\right) / 2\right)}{\left(y_{n}-x_{n}\right)}\right| \\
& \quad<\left|\frac{\sin \left(\left(y_{n}^{\prime}+x_{n}^{\prime}\right) / 2\right) \sin \left(\left(y_{n}^{\prime}-x_{n}^{\prime}\right) / 2\right)}{\left(y_{n}^{\prime}-x_{n}^{\prime}\right)}\right| .
\end{aligned}
$$

Thus $P_{2}<0$ for $P_{1}=0$.

So if by the first condition of (13) $\sum_{j} P_{j}=0$ then the second condition of (13) cannot be met.

Let us now give the alternative proof.

Proof. Consider the first condition of (13). Then integration by parts and symmetry of $f$ with respect to $\pi / 2$ yield $C^{\prime}=$ $\int_{0}^{2 \pi} f(t) d t=\left.f(t)(t-\pi / 2)\right|_{0} ^{2 \pi}-\int_{0}^{2 \pi} f^{\prime}(t)(t-\pi / 2) d t=$ $-\int_{0}^{2 \pi} f^{\prime}(t)(t-\pi / 2) d t$.

Similarly the two latter conditions of (13) turn into relations $K=\int_{0}^{2 \pi} f^{\prime}(t) \cos (t) d t$ and $0=\int_{0}^{2 \pi} f^{\prime}(t) \sin (t) d t$, respectively.

Thus we have reduced the problem to one similar to the one already solved with one modified condition.

Again as in the proof of the first statement we can show that the solution is a linear combination of Dirac $\delta$-functions.

We have to minimize the functional

$$
\sum \alpha_{i}
$$

under the following conditions: $\alpha_{i} \leq 0, \sum \alpha_{i}\left(x_{i}-\pi / 2\right)=-C^{\prime}$, $\sum \alpha_{i} \cos \left(x_{i}\right)=K$ on the interval $x_{i} \in[\pi / 2,3 \pi / 2]$. The last condition may be rewritten as follows: $\sum \alpha_{i} y_{i}=-C^{\prime}$, $\sum \alpha_{i} \sin \left(y_{i}\right)=-K, y_{i} \in[0, \pi]$.

Let us first prove that we may assume that $y_{i} \in[\pi / 2, \pi]$. Let $y_{1}<\pi / 2$. Consider $f^{\prime}=\alpha_{1} \delta_{\pi-y_{1}}+\alpha_{2} \delta_{\pi / 2}$. Then the equation system

$$
\begin{aligned}
& \alpha_{0} y_{1}=\alpha_{1}\left(\pi-y_{1}\right)+\frac{\alpha_{2} \pi}{2}, \\
& \alpha_{0} y_{1}=\alpha_{1} \sin \left(\pi-y_{1}\right)+\alpha_{2}
\end{aligned}
$$

is uniquely resolvable. Moreover $\left|\alpha_{1}\right|+\left|\alpha_{2}\right|<\left|\alpha_{0}\right|$. That is, if we replace $\alpha_{0} \delta_{y_{1}}$ with $\alpha_{1} \delta_{\pi-y_{1}}+\alpha_{2} \delta_{\pi / 2}$ then we obtain the function with smaller value of the functional. Hence all the points $y_{i}$ are greater than $\pi / 2$.

We next apply the Lagrange multipliers method. We have the function

$$
\begin{aligned}
F\left(\alpha_{i}, y_{i}\right)= & \sum_{i=1}^{n} \alpha_{i}-\lambda\left(\sum_{i=1}^{n} \alpha_{i} y_{i}+C^{\prime}\right) \\
& -\mu\left(\sum_{i=1}^{n} \alpha_{i} \sin \left(y_{i}\right)+K\right) .
\end{aligned}
$$

The derivatives of this function with respect to $y_{i}$ generate the equation system $\lambda+\mu \cos \left(y_{i}\right)=0, i=1, \ldots, n$, which 
possesses the unique and common solution $y_{0}$ on the given interval. Moreover $\lambda, \mu$ are of the same sign. Also they are both nonnegative since otherwise there is no solution of the equation which is a derivative of $F$ with respect to $\alpha_{i}: 1-\lambda y_{i}-$ $\mu \sin \left(y_{i}\right)=0$.

The principle minors of the second derivative matrix are equal to $\mu \sin (y),(\lambda+\mu \cos (y))^{2} / 4, y^{2} \mu \sin (y) / 4$, and $\alpha^{2}(\sin (y)-y \cos (y))^{2} / 16$, so all of them are positive. Hence $F$ is minimal at the point $y_{0}$.

The last proof allows us to find a series of solutions minimizing functionals of the form

$$
L(1, n)(f)=\int_{0}^{2 \pi}\left|f^{(n)}(t)\right| d t .
$$

The first condition of (13) turns into

$$
C^{\prime}=(-1)^{n} \int_{0}^{2 \pi} f^{(n)}(t) p_{n}\left(t-\frac{\pi}{2}\right) d t .
$$

The second condition of (13) becomes

$$
K^{\prime}=\int_{0}^{2 \pi} f^{(n)}(t) \sin \left(t+\frac{n \pi}{2}\right) d t .
$$

Finally the third condition of (13) transforms into

$$
0=\int_{0}^{2 \pi} f^{(n)}(t) \cos \left(t+\frac{n \pi}{2}\right) d t
$$

Here the polynomial $p_{n}(t-\pi / 2)$ on $[-\pi / 2,3 \pi / 2]$ is such that $\int p_{n}^{(k)}(t-\pi / 2) d t=0, k=0, n-1$. So, for $n=1, p_{1}=t-\pi / 2$, and for $n=2, p_{2}=(t-\pi / 2)^{2}-\pi^{2} / 3$, and so on.

Now induction on $n$ allows us to prove the following facts:

(1) Case of $n=2 k, k \in \mathbb{N}$ : the polynomial $p_{n}$ is even with respect to $\pi / 2$ and monotone on $[\pi / 2,3 \pi / 2]$.

(2) Case of $n=2 k+1, k \in \mathbb{N}$ : the polynomial $p_{n}$ is odd with respect to the point $(\pi / 2,0)$ and is either strictly convex or concave on $[\pi / 2,3 \pi / 2]$.

The induction base is $n=1,2$.

Hence the solution is again a sum of two Dirac $\delta$ functions. This is clear for even $n$ since there exist only two common extremal points for $p_{n}$ and $\sin (t)$.

For the case of the odd $n$ this fact follows from strict convexity or concavity of the polynomial $p_{n}$ and the function $\cos (t)$ graph on $[\pi / 2,3 \pi / 2]$. Thus for $a \delta_{\alpha}+b \delta_{\beta}$ we obtain $a+b>c$ for $c \delta_{(\alpha+\beta) / 2}$.

Let us now write down the solution representation for the functional $L(1, n)(f)$.

The statement $s$ given above shows us that the $n$th derivative of the solution $f$ is a linear combination of Dirac $\delta$-functions. Hence $f$ is a piecewise polynomial function.

Assume that $n=2 k$. Then

$F_{1}(t)$

$$
= \begin{cases}\sum_{j=1}^{n-2} P_{j}\left(\left(t-\frac{\pi}{2}\right) \bmod 2 \pi\right)^{j}, & t \in[0, \pi+\alpha] \bigcup[2 \pi-\alpha, 2 \pi] \\ \sum_{j=1}^{n-2} Q_{j}\left(t-\frac{3 \pi}{2}\right)^{j}, & t \in(\pi+\alpha, 2 \pi-\alpha) .\end{cases}
$$

Here $P_{j}, Q_{j}$, and $\alpha$ are such that (1) $f_{+}^{l}(\pi+\alpha)=f_{-}^{l}(\pi+\alpha)=$ $-f_{+}^{l}(2 \pi-\alpha)=-f_{-}^{l}(2 \pi-\alpha), l=0, \ldots, n-3$.

(2) Conditions (13) hold.

Assume that $n=2 k-1$. Then

$$
\begin{aligned}
& F_{1}(t) \\
& = \begin{cases}\sum_{j=0}^{n-2} P_{j}\left(\left(t-\frac{\pi}{2}\right) \bmod 2 \pi\right)^{j}, & t \in\left[\frac{\pi}{2}, \frac{3 \pi}{2}\right] \\
\sum_{j=0}^{n-2} Q_{j}\left(t-\frac{3 \pi}{2}\right)^{j}, & t \in\left(0, \frac{\pi}{2}\right) \bigcup\left(\frac{3 \pi}{2}, 2 \pi\right] .\end{cases}
\end{aligned}
$$

Here $P_{j}, Q_{j}$ are such that

(1) $\begin{aligned} & f_{+}^{l}(\pi / 2)= \\ & 0, \ldots, n-3 ;\end{aligned} \quad f_{-}^{l}(\pi / 2), f_{+}^{l}(3 \pi / 2)=f_{-}^{l}(3 \pi / 2), l=$
$0.1=$

(2) conditions (13) hold.

\section{Example}

The contour reconstruction procedure is as follows.

Since conditions (13) describe the Fourier coefficients of the velocity logarithm decomposition we construct the contours considering the first 60 (this number is by no means fixed and simply provided satisfactory profile shapes) terms of this series. Then we modify these terms with the help of the optimization problems solutions and reconstruct the discrete sets of the respective contours points. The author considered only 100 points so 200 numerical integrals had to be calculated for each contour. All the calculations here were performed in the Computer Algebra System Maxima.

Let us consider the solutions for the norms $\|F\|_{2},\|F\|_{\infty}+$ $1 / 2\left\|F^{\prime}\right\|_{\infty}$ and the functional $L(1,3)(F)$. In order to minimize the last functional we must find the solution for the following system on $a, b, c$, and $d$ :

$$
\begin{aligned}
& \frac{4}{3}\left(\frac{b^{3 / 2}}{a^{1 / 2}}-\frac{d^{3 / 2}}{c^{1 / 2}}\right)=K, \\
& a b=c d \\
& \sqrt{\frac{b}{a}}=\pi-\sqrt{\frac{d}{c}} \\
& 4\left(a\left(\sin \left(\sqrt{\frac{b}{a}}\right)-\sqrt{\frac{b}{a}} \cos \left(\sqrt{\frac{b}{a}}\right)\right)\right. \\
& \left.\quad+c\left(\sin \left(\sqrt{\frac{d}{c}}\right)-\sqrt{\frac{d}{c}} \cos \left(\sqrt{\frac{d}{c}}\right)\right)\right)=C^{\prime} .
\end{aligned}
$$

So we have the piecewise polynomial function

$F(x)$

$$
=\left\{\begin{array}{l}
-a\left(x-\frac{\pi}{2}\right)^{2}+b, \quad x \in\left[\frac{\pi}{2}-\sqrt{\frac{b}{a}}, \frac{\pi}{2}+\sqrt{\frac{b}{a}}\right] \\
c\left(x-\frac{3 \pi}{2}\right)^{2}-d, \quad x \in\left[\frac{3 \pi}{2}-\sqrt{\frac{c}{d}}, \frac{\pi}{2}+\sqrt{\frac{c}{d}}\right] .
\end{array}\right.
$$




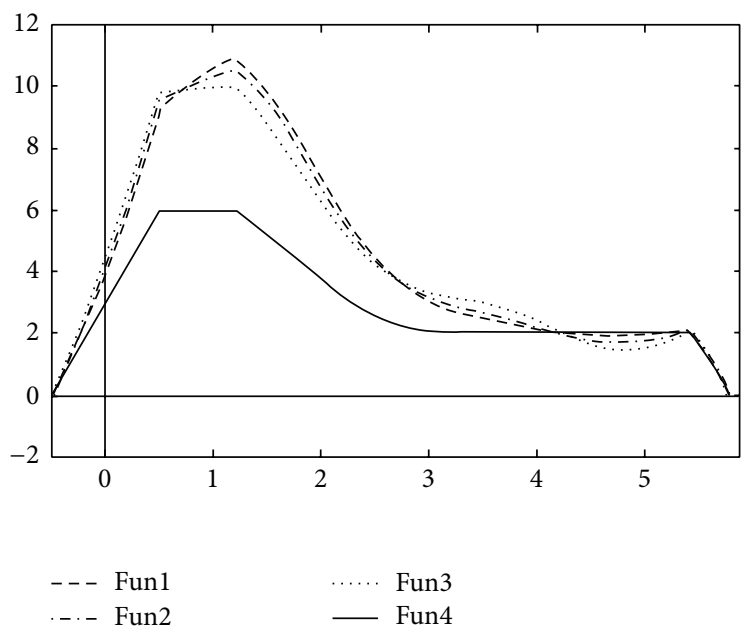

FIGURE 2: Fun1: the velocity distribution generated by the norm $\|F\|_{2}$. Fun2: the velocity distribution generated by the functional $L(1,3)$. Fun3: the velocity distribution generated by the norm $\|F\|_{\infty}+1 / 2\left\|F^{\prime}\right\|_{\infty}$. Fun 4 : the initial velocity distribution.

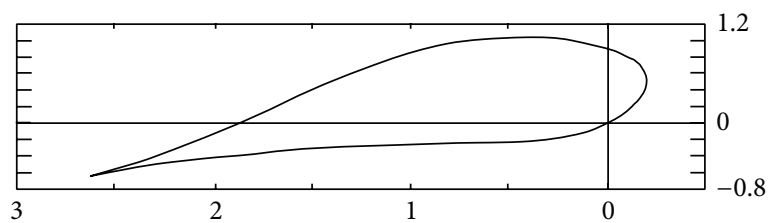

(a)

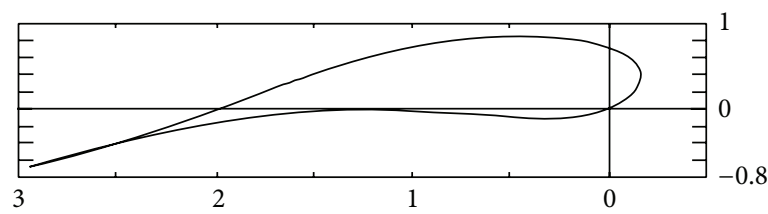

(c)

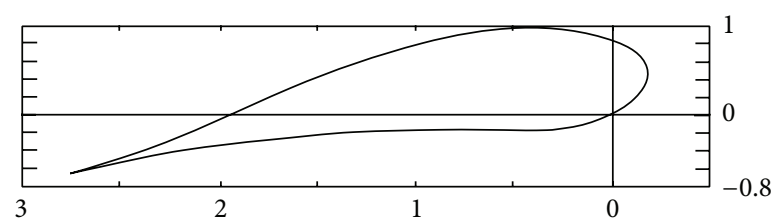

(b)

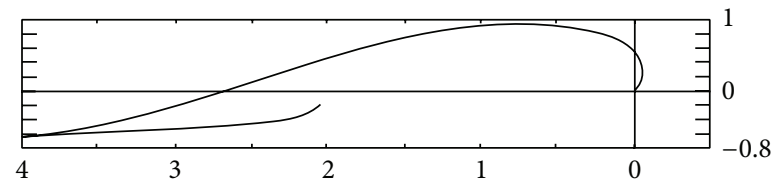

(d)

FiguRE 3: (a) The contour corresponding to $\|F\|_{2}$. (b) The contour corresponding to $L(1,3)$. (c) The contour corresponding to $\|F\|_{\infty}+1 / 2\left\|F^{\prime}\right\|_{\infty}$. (d) The contour corresponding to the initial velocity distribution.

Figure 2 shows velocity distributions for the norms $\|F\|_{2}$, $\|F\|_{\infty}+1 / 2\left\|F^{\prime}\right\|_{\infty}$ and the functional $L(1,3)$. The integral norm solutions are better in majority of points but in certain points the norm $\|F\|_{\infty}+1 / 2\left\|F^{\prime}\right\|_{\infty}$ is the best possible one. This is true for all of the examples constructed by the author. Since the velocity distribution describes the contour these differences result in rather palpable differences in contours presented in Figure 3. Also direct calculations tell us that the boundary layer thickness is minimal in the case of the norm $\|F\|_{\infty}+1 / 2\left\|F^{\prime}\right\|_{\infty}$ and maximal in the case of the functional $L(1,3)$. Thus the drag (that is wind or fluid resistance) is also the smallest for the norm $\|F\|_{\infty}+1 / 2\left\|F^{\prime}\right\|_{\infty}$.

\section{Norm in the Space $L^{p}$}

Let us find the function with minimal norm $\|f\|_{L^{p}}$ in the space of all functions integrable on $[0,2 \pi]$ and meeting conditions (13).
The problem then can be resolved by methods of calculus of variations.

The relative Euler-Lagrange function is

$$
\begin{aligned}
& \int_{0}^{2 \pi}|f(t)|^{p} d t-\lambda\left(\int_{0}^{2 \pi} f(t) \sin (t) d t-C^{\prime}\right) \\
& \quad-\mu\left(\int_{0}^{2 \pi} f(t) d t-K\right) .
\end{aligned}
$$

Hence

$f(t)$

$$
= \begin{cases}\frac{1}{p}(\lambda \sin (t)+\mu)^{1 /(p-1)}, & x \in[-\alpha, \pi+\alpha] \\ -\frac{1}{p}(\lambda \sin (t)+\mu)^{1 /(p-1)}, & x \in[\pi+\alpha, 2 \pi-\alpha] .\end{cases}
$$


Here $\alpha, \lambda$, and $\mu$ are solutions of system

$$
\begin{aligned}
& \int_{-\alpha}^{\pi+\alpha}(\lambda \sin (t)+\mu)^{1 /(p-1)} \sin (t) d t \\
& \quad-\int_{\pi+\alpha}^{2 \pi-\alpha}(\lambda \sin (t)+\mu)^{1 /(p-1)} \sin (t) d t=p C^{\prime}, \\
& \int_{-\alpha}^{\pi+\alpha}(\lambda \sin (t)+\mu)^{1 /(p-1)} d t \\
& \quad-\int_{\pi+\alpha}^{2 \pi-\alpha}(\lambda \sin (t)+\mu)^{1 /(p-1)} d t=p K, \\
& \lambda \sin \alpha-\mu=0 .
\end{aligned}
$$

\section{Conflict of Interests}

The author declares that there is no conflict of interests regarding the publication of this paper.

\section{References}

[1] P. N. Ivan'shin, "Quasisolutions of one inverse boundary-value problem of aerohydromechanics," Russian Mathematics, vol. 57, no. 5, pp. 9-19, 2013.

[2] A. N. Kolmogorov and S. V. Fomin, Elements of the Theory of Functions and Functional Analysis, vol. 1, Courier Dover Publications, 1999.

[3] F. G. Avkhadiev and D. V. Maklakov, "A criterion for the solvability of the problem of constructing wing profiles based on a cavitations diagram," Russian Mathematics, vol. 38, no. 7, pp. 1-10, 1994.

[4] D. F. Abzalilov, E. V. Varsegova, and N. B. Il'inskii, "Lift maximization for a smooth contour placed in ow with a vortex," Computational Mathematics and Mathematical Physics, vol. 50, no. 10, pp. 1760-1766, 2010.

[5] P. N. Ivan'shin, "Methods of constructing quasisolutions in inverse boundary-value aerohydrodynamics problems," Russian Aeronautics, vol. 57, no. 3, pp. 253-259, 2014.

[6] V. K. Ivanov, "Conditions for well-posedness in the Hadamard sense in spaces of generalized functions," Siberian Mathematical Journal, vol. 28, no. 6, pp. 906-911, 1987.

[7] A. M. Elizarov, "Quasisolutions of an exterior inverse boundary value problem," Soviet Mathematics, vol. 28, no. 10, pp. 54-65, 1984.

[8] A. M. Elizarov and D. A. Fokin, "Exact solutions to the principal variational inverse boundary value problem of aerohydrodynamics," Doklady Physics, vol. 1, no. 11, pp. 673-679, 2004.

[9] L. A. Aksent'ev, N. B. Il'inskii, M. T. Nuzhin, R. B. Salimov, and G. G. Tumashev, "The theory of inverse boundary problems for analytic functions and its applications," Journal of Soviet Mathematics, vol. 18, no. 4, pp. 479-515, 1982.

[10] A. M. Elizarov, D. A. Fokin, and M. S. Galyavieva, "Problems of hydrofoil design for a given range of angles of attack," Zeitschrift für Angewandte Mathematik und Mechanik, vol. 76, no. 6, pp. 337-340, 1996.

[11] F. D. Gahov, Boundary Value Problems, Pergamon Press, 1966.

[12] W. Mangler, "The lift distribution of wings with end plates," NACA TM 856, 1938. 


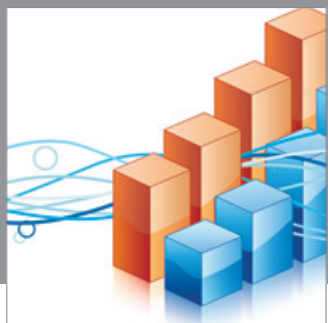

Advances in

Operations Research

mansans

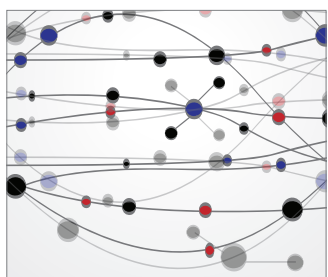

The Scientific World Journal
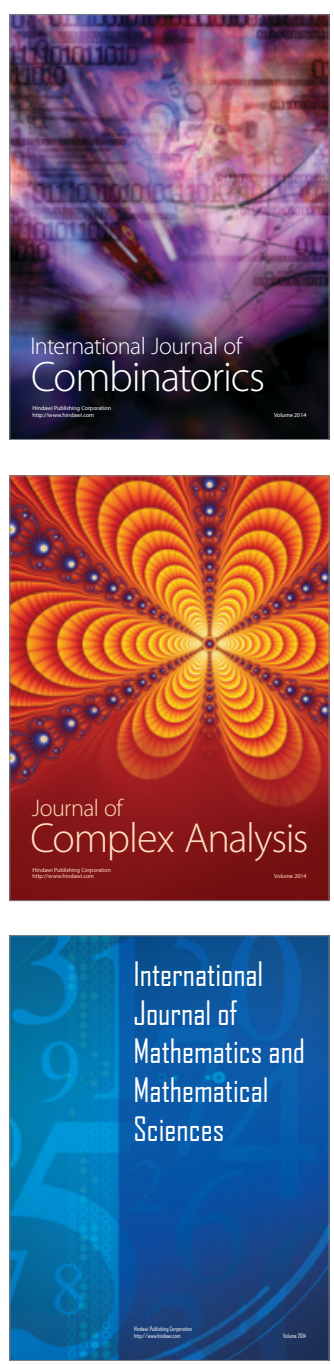
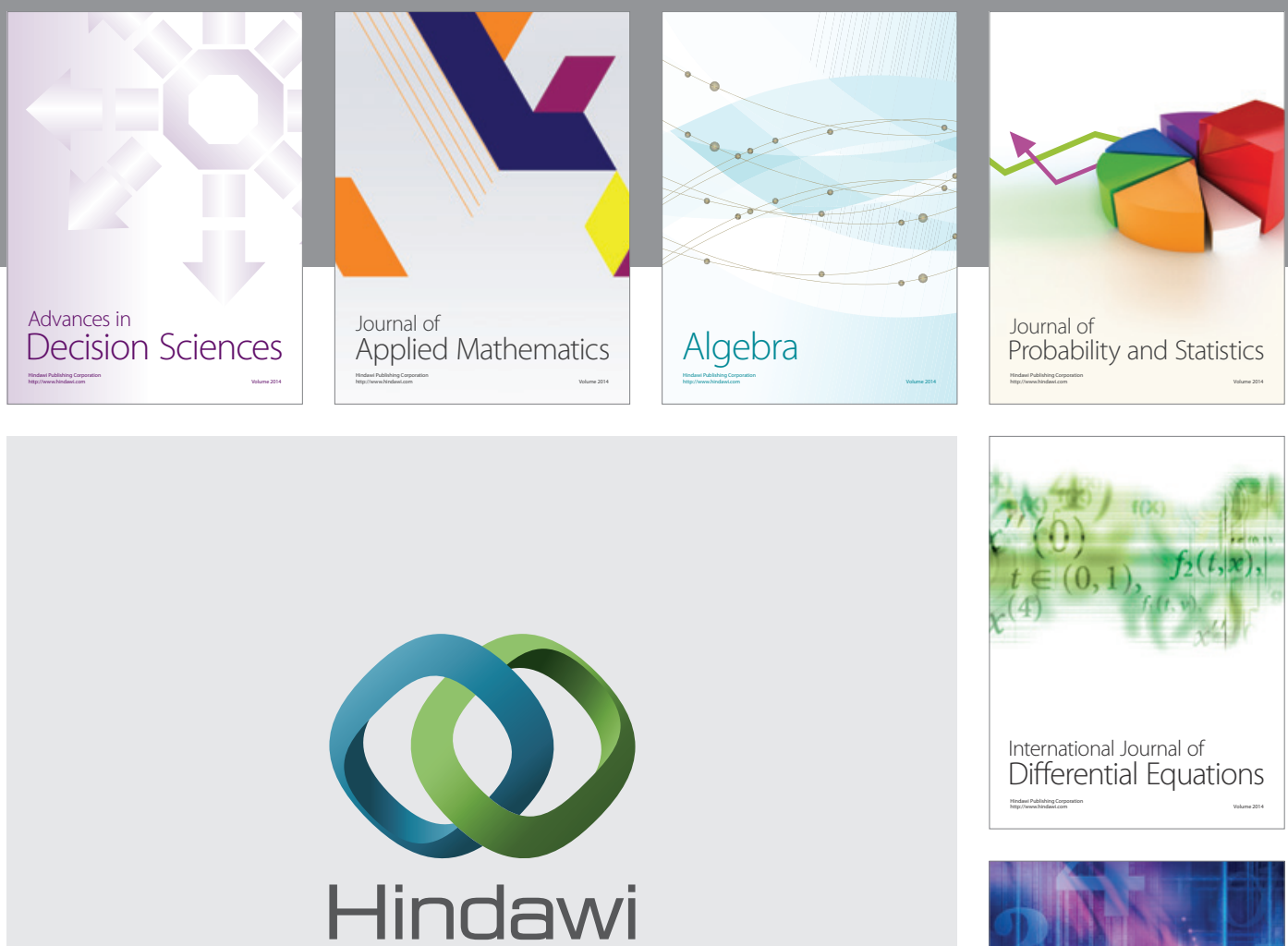

Submit your manuscripts at http://www.hindawi.com
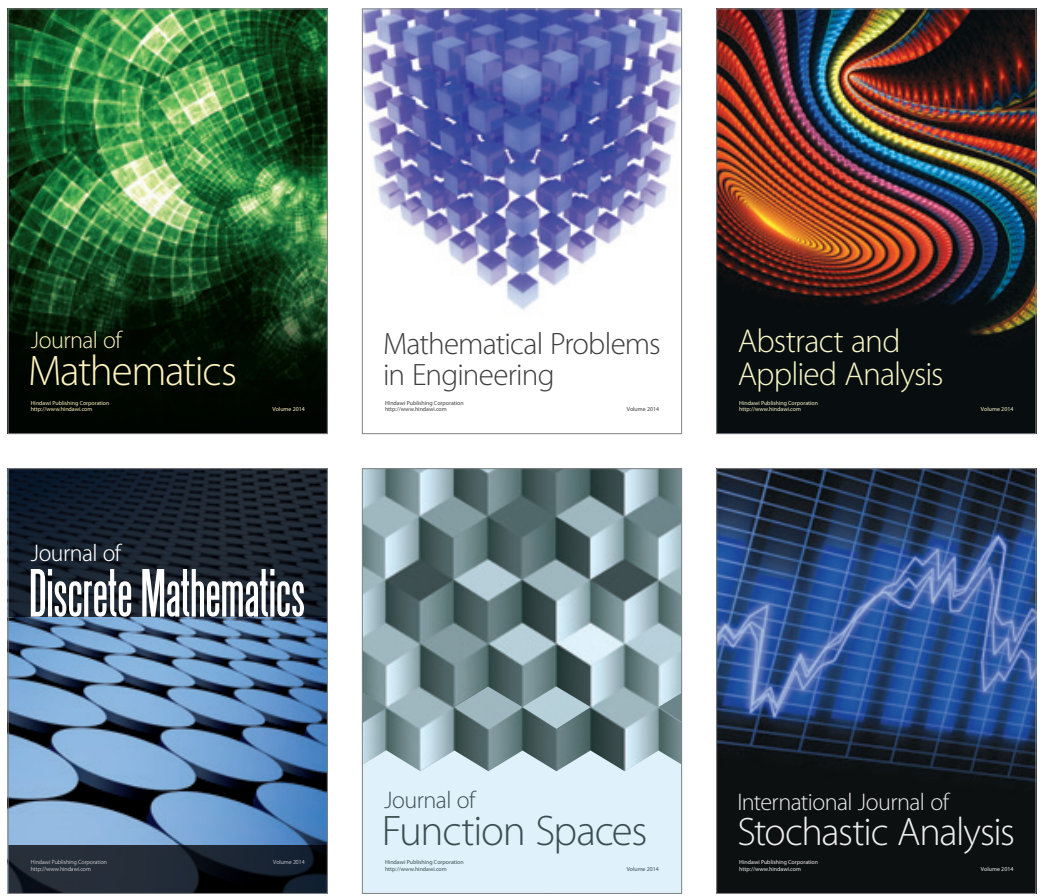

Journal of

Function Spaces

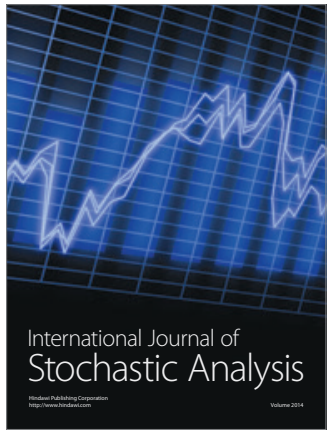

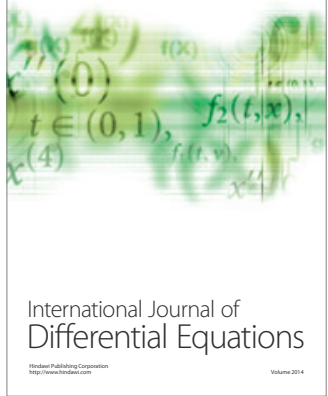
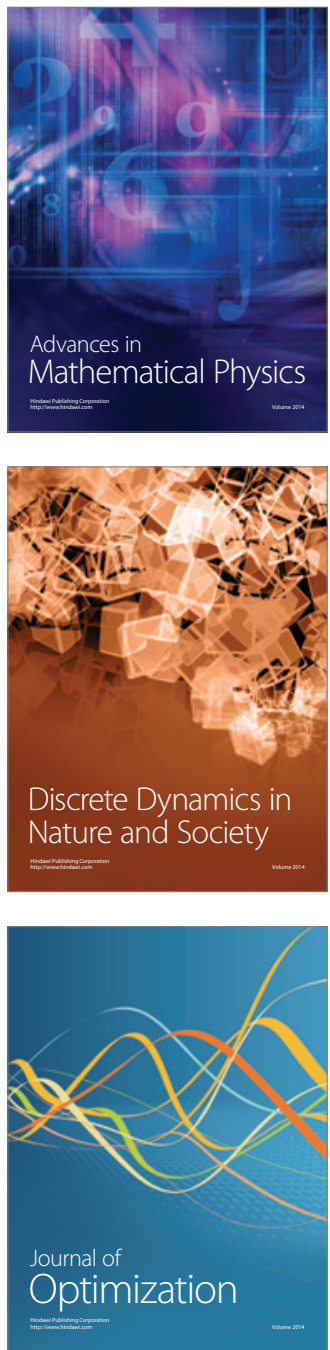